\section{FOCUS CONTENTS}

237 Combining immunotherapy and targeted therapies in cancer treatment Matthew Vanneman and Glenn Dranoff

252 The blockade of immune checkpoints in cancer immunotherapy Drew M. Pardoll

265 Cancer immunotherapy via dendritic cells Karolina Palucka and Jacques Banchereau

278 Antibody therapy of cancer

Andrew M. Scott, Jedd D. Wolchok and Lloyd J. Old

289 OPINION Unmasking the immune recognition of prostate cancer with CTLA4 blockade Serena S. Kwek, Edward Cha and Lawrence Fong

298 OPINION The immune contexture in human tumours: impact on clinical outcome Wolf Herman Fridman, Franck Pagès, Catherine Sautès-Fridman and Jérôme Galon

307 VIEWPOINT The determinants of tumour immunogenicity Thomas Blankenstein, Pierre G. Coulie, Eli Gilboa and Elizabeth M. Jaffee

Gemma K. Alderton, Senior Editor, Nature Reviews Cancer

\title{
Tumour immunotherapy - leukocytes take up the fight
}

\section{Gemma K. Alderton and Yvonne Bordon}

Abstract | The immune system can promote the elimination of tumours, but often immune responses are modulated or suppressed by the tumour microenvironment. In this joint Focus from Nature Reviews Cancer and Nature Reviews Immunology, leading researchers describe our current understanding of the complex interactions that occur between the immune system and tumour cells, and discuss how the power of the immune system can be harnessed by anticancer immunotherapies.

Since the use of Coley's toxins for the treatment of cancer in the late nineteenth century, scientists have been aware of the potential of the immune system to provide enduring protection from cancer. However, often the immunogenicity of a tumour is variable and the challenge for researchers has been to determine why tumour cells tend not to induce a robust anti-tumour immune response.

It is clear that there are several strategies by which tumour cells evade an immune response and, encouragingly, some of these have been successfully targeted to result in durable anti-tumour immune responses. For example, inhibitory signalling pathways that restrict immune cell activation (known as immune 'checkpoints') can be inhibited and such an approach has been shown to induce regression of melanomas and other types of cancer. The exciting progress in targeting immune checkpoints in general is discussed by Drew Pardoll, and targeting an immune checkpoint in prostate cancer is described by Lawrence Fong and colleagues. Further encouraging progress in targeting the immune response is discussed by Matthew Vanneman and Glenn Dranoff, who highlight how oncologists have inadvertently been using immunotherapy for some time. They outline the mechanisms by which various anticancer therapies induce an immune response that contributes to their efficacy. Understanding how the immune system is affected by different types of therapy could inform our choices regarding combining immunotherapies to improve anti-tumour immune responses. Similarly, understanding cancer serology and the behaviour of monoclonal antibodies in patients with cancer, as discussed by Andrew Scott and colleagues, can also help us in understanding how to refine therapeutic antibodies to ensure tumour cell killing.

Several articles in this Focus concentrate on how different immune cell populations function in, and are shaped by, the tumour microenvironment. Eric Vivier and colleagues describe how natural killer (NK) cells and NKT cells can recognize tumour cells and promote their elimination. An increased understanding of the molecular interactions involved in this process has led to the development of therapies that can boost the antitumour activities of NK cells and NKT cells in patients with cancer. Myeloid cells, such as dendritic cells (DCs) and macrophages, can also drive potent anti-tumour immune responses. Indeed, Karolina Palucka and Jacques Banchereau review the use of DC-based therapies to treat cancer. Conversely, Dmitry Gabrilovich and colleagues discuss how tumour cells inhibit the beneficial tumoursuppressive effects of the myeloid system and instead promote the development of immunosuppressive myeloid cells, which favour tumour growth.

Nicholas Restifo and colleagues focus on how effector T cells detect and destroy tumour cells. In vitro proliferation and re-infusion of a patient's own tumour-specific T cells has already shown success in treating some types of cancer. The type and distribution of immune cells - and of the cytokines and chemokines that regulate them - within the tumour microenvironment can determine the extent of immunomodulation and hence the prognosis of patients with cancer, as discussed by Jérôme Galon and colleagues.

Of course, many issues remain to be resolved in the ever-expanding field of cancer immunology. In particular, what determines the immunogenicity of a tumour? The Viewpoint article contains the thoughts of four experts in response to this important question. It is clear that the continued success of anticancer immunotherapy will rely on close collaboration between experts from the fields of cancer and immunology.

All of the Focus articles can be accessed for free until the end of September 2012 at http://www.nature.com/ reviews/focus/tumourimmunology thanks to support from Boehringer Ingelheim. 